\title{
is Research Square \\ Endothelium Response to Iron Sucrose Nanoparticles in Static Versus Dynamic Culture Model
}

\section{Niusha Nikravesh}

Swiss Federal Laboratories for Materials Science and Technology

\section{Liliane Diener}

Swiss Federal Laboratories for Materials Science and Technology

\section{Savvina Chortarea}

Swiss Federal Laboratories for Materials Science and Technology

\section{Alexandra Rippl}

Swiss Federal Laboratories for Materials Science and Technology

Peter Wick ( $\nabla$ peter.wick@empa.ch )

Swiss Federal Laboratories for Materials Science and Technology

\section{Research Article}

Keywords: nanomedicines, endothelial cells, dynamic, iron sucrose

Posted Date: March 15th, 2021

DOI: https://doi.org/10.21203/rs.3.rs-279067/v1

License: (c) (1) This work is licensed under a Creative Commons Attribution 4.0 International License. Read Full License 


\section{Abstract}

Nanomedicines are promising therapeutic compounds allowing the development of new treatment approaches. However, important factors affecting the behavior of nanoparticles in vivo cannot be simulated in conventional static models. Dynamic cell cultures, where cells are cultivated in the presence of shear stress, have the potential to bridge this gap by mimicking critical features of physiological conditions. Iron-carbohydrate nanoparticles are a dominant treatment for iron deficiency unresponsive to oral iron supplements. Compared to the data available from clinical studies, little is known about the interaction of these particles with endothelial cells. Our approach implements and compares a microchannel-based dynamic human endothelium model to the static culture to explore potential differences in cells response after exposure to iron nanoparticles. Differences in cellular uptake are observable using Prussian blue assay. There is a noticeable increase on VCAM- 1 and ICAM-1 gene expression on endothelial cells activated by inflammatory responses, in cells exposed to nanoparticles under static condition. Results show that cytotoxicity caused by iron particles is significantly lower under dynamic condition compared to static cultures. We demonstrate that inclusion of dynamic flow and biological fluids are positive steps towards nanoparticle evaluation in a physiologically relevant in vitro model.

\section{Introduction}

Despite the broad range of nanomaterials employed for non-biomedical applications, the progress in integrating nanotechnology into medicine has been relatively limited. Up until now, several numbers of approved therapeutic nanomedicine products, such as Doxil (liposomal infusion), Onivyde (irinotecan liposome injection), Abraxane (paclitaxel protein-bound), and iron nanomedicines (polynuclear iron (III)hydroxide cores surrounded by carbohydrate shells) are commercially available. ${ }^{[1]-[4]}$ However, there is a limited understanding of the complex pharmacodynamics of both investigational and approved nanomedicines, and there are several potential explanations for this limitation. Firstly, considerable disparity exists concerning the biological impacts of therapeutic nanoparticles. Such weak correlations between the results from in vitro, in vivo, and clinical studies make it challenging to draw a conclusion over the safety and effectiveness of a particular nanomedicine product. ${ }^{[5],[6]}$ Further, extensive knowledge of interactions between nanoparticles and cells, known as nano-bio interfaces, is not yet fully available, and this can lead to data misinterpretation in literature. ${ }^{[7]}$ Finally, current in vitro assays and cell-based platforms have mostly been introduced and employed far behind the advent of nanotechnology; accordingly, they might not be considered to be fully compatible with nanomedicine related research. Lack of comprehensive and realistic in vitro models can hinder accurate prediction of biological readouts for the development of nanotherapeutic compounds. ${ }^{[7],[8]}$

Over the past years, more realistic in vitro models using primary cells have been developed for a detailed investigation into nanoparticles interaction with cells. Although the application of primary cell models can retain critical features, they cannot fully imitate the in vivo environment. Considering the nature of 
different cell types, several requirements should be fulfilled to achieve physiologically relevant in vitro models. For cells under permanent dynamic condition, such as endothelial cells, the cellular phenotype is markedly influenced by shear stress and culture condition. ${ }^{[8],[9]}$ Advanced in vitro systems that can mimic the interactions of endothelial cells with nanoparticles in conditions with high physiological relevance should be considered to increase the accuracy of prediction.

Endothelial cells line the interior surface of the vasculature covering a maximum surface area of $7000 \mathrm{~m}^{2}$ in an adult . ${ }^{[10]}$ The pivotal function of the endothelium, which represents the cellular interface between circulating blood and the underlying tissue, is the maintenance of permeability across the vessel wall by controlling the flow of nutrients, bioactive molecules, blood and immune cells within the circulatory system. Besides, these cells are majorly involved in the regulation of vascular haemostasis as well as on inflammatory and immune responses. ${ }^{[10]-[12]}$ Endothelial cells are continuously exposed to shear forces as a result of the blood flow, and these mechanical forces have been shown to play an essential role in regulating endothelial cell structure and function. ${ }^{[8],[13]}$ The importance of the effect of flow on these cells has been mostly assessed in cell-based models for characterizing endothelial cell biology and pathophysiology, for instance, in the field of atherosclerosis research. ${ }^{[11],[13]}$

Given the importance of realistic in vitro experimental models and the reproducibility of cell culture systems, the focus of the present study is the application of a semi-automated perfusion system to achieve a mature endothelial model. Utilizing a microfluidic 3D approach to mimic the normal physiological conditions of quiescent endothelial cells, we investigated the in vitro interaction between iron sucrose nanoparticles and primary human endothelial cells. Here, we used intravenous iron sucrose nanoparticles to evaluate potential cytotoxicity and uptake behavior of cells under static versus dynamic culture condition. Iron carbohydrate complexes are within a category of medicinal products, known as non-biological complex drugs. Amongst various intravenous iron carbohydrate complexes, iron sucrose is the most widely used representative of intravenous nanomedicine product for the treatment of iron deficiency with favorable clinical outcomes. ${ }^{[14]}$ Based on current knowledge, little is known about interactions between iron-carbohydrate complexes and the vascular cells, and some contradicting reports regarding endothelium toxicity of iron sucrose in static cell cultures have been published in recent years. [15], [16] In the investigation presented here, we explore if a more physiological condition in the form of biochemical stress will affect the outcomes of nanoparticles assessment and whether the interaction of nanoparticles with cells under dynamic culture condition can exhibit significant differences in biological responseswhen compared with the conventional static endothelial models.

\section{Materials And Method}

\subsection{Cell culture}

Primary human umbilical vein endothelial cells (HUVECs) were purchased from Lonza (Switzerland) and cultured in complete endothelium cell growth medium (ECGM, PromoCell, Germany) containing: $2 \%$ fetal 
calf serum (FCS), $90 \mu \mathrm{g} \mathrm{mL}^{-1}$ heparin, $0.1 \mathrm{ng} \mathrm{mL}^{-1}$ epidermal growth factor, $1 \mathrm{ng} \mathrm{mL}^{-1}$ basic fibroblast growth factor, $1 \mu \mathrm{g} \mathrm{mL}^{-1}$ hydrocortisone, and $0.4 \%$ endothelial cell growth supplement. All cultures were incubated in T-75 flasks coated with $1 \%$ gelatine (Sigma, Germany) at $37^{\circ} \mathrm{C}$ with $5 \% \mathrm{CO}_{2}$ until they reached $80 \%$ confluency. Throughout this study, HUVECs in the passage number of 1-6 were used, and the culture medium was changed three times a week. For experimental purposes, cells were detached from the flask using Accutase (StemCell Technology, Canada) followed by centrifugation at $200 \mathrm{xg}$ for 5 $\min$.

\subsection{Treating cells with iron-carbohydrate complexes}

Iron sucrose preparations (Venofer) were kindly provided by Vifor Pharma, (St. Gallen, Switzerland). Based on clinical observations after administration of $100 \mathrm{mg}$ of intravenous iron sucrose, the expected plasma iron concentration is close to $600 \mu \mathrm{M}$. In clinical studies with peritoneal dialysis patients, higher concentrations of iron sucrose preparations up to $300 \mathrm{mg}$ have been used, thus a plasma iron concentration of $1800 \mu \mathrm{M}$ can be expected. ${ }^{[17]}$ In our studies, all in vitro concentrations were calculated according to the highest clinically relevant plasma iron concentration (iron concentration of $1800 \mu \mathrm{M}$ ).

\subsection{Static experimental setup}

$\mu$-slide I luer ${ }^{0.4}$ (Ibidi, Germany) with a growth area of $2.5 \mathrm{~cm}^{2}$, total channel volume of $100 \mu \mathrm{L}$, and two reservoirs (each with the capacity of $60 \mu \mathrm{L}$ ) were used for all the experimental setups. In each $\mu$-slide, the medium added into reservoirs flows to the growth channel by capillary forces. In order to enhance cells attachment, $\mu$-slides were first coated with $75 \mu \mathrm{g} / \mathrm{cm}^{2}$ collagen IV (Sigma, Germany). The slides were then seeded with primary HUVECs at a concentration of $1.5 \times 10^{6} \mathrm{cells} / \mathrm{mL}$. All experiments were performed based on conventional static experimental designs. Briefly, cells were cultured for two days at $37^{\circ} \mathrm{C}$ with $5 \% \mathrm{CO}_{2}$, and the culture medium was refreshed twice a day to maintain cell viability. After two days of cultivation, cells were incubated in complete medium containing $1800 \mu \mathrm{M}$ of iron sucrose for another 24 h. Control samples were prepared in the absence of iron nanomedicine.

\subsection{Experimental setup for dynamic culture}

- For cell culture under flow, HUVECs were seeded in the $\mu$-slides as described above. After incubation of cells for $24 \mathrm{~h}$, the $\mu$-slides were connected to a perfusion system (Ibidi, Germany) with a closedloop. The perfusion sets were then connected to an air pressure pump, by which the precise control of flow rate and shear stress was achieved using the PumpControl software. Cell monolayer inside the channel was then perfused with complete medium under unidirectional flow at a shear stress of 10 dyne $/ \mathrm{cm}^{2}$ in order to obtain shear stress that can closely resemble the physiological situation with HUVECs (Fig. 1). After preliminary observation, a cultivation time of two weeks was chosen to achieve mature quiescent endothelium. During this time, the cell culture medium was refreshed two times, while each time only half of the reservoir was replaced with fresh medium ( $5 \mathrm{~mL})$. On day 14 , the culture medium was replaced with complete culture medium containing $1800 \mu \mathrm{M}$ of iron sucrose, 
and cells were subjected to shear force for another $24 \mathrm{~h}$. For further experiments, $\mu$-slides were detached from the perfusion sets and cells were examined accordingly.

\subsection{Endothelial cell viability}

The viability of HUVECs under dynamic condition was assessed using Alamar Blue cell proliferation assay (Thermo Fisher Scientific, Germany). During cellular metabolism, the assay reagent reduces from a non-fluorescence blue coloured agent to a fluorescent red substance, where the amount of assay reagent reduction is proportional to the cell number. After washing several times the cells with PBS (containing $\mathrm{Mg}^{2+}$ and $\left.\mathrm{Ca}^{2+}\right)$, HUVECs were exposed to Alamar Blue reagent in complete cell culture medium $(1: 10 \mathrm{v} / \mathrm{v}$ ratio). Samples were incubated in the dark for $4 \mathrm{~h}$ at $37^{\circ} \mathrm{C}$. The fluorescence was then measured (590 $\mathrm{nm}$ emission and $560 \mathrm{~nm}$ excitation) using a microplate reader (Mithras LB 940 multimode microplate Reader, Berthold technology, Switzerland).

\subsection{Immunofluorescence staining and image quantification}

HUVECs were pre-fixed for 2 min by adding $60 \mu \mathrm{L}$ of $4 \%$ paraformaldehyde solution (PFA, Sigma, Germany) to the culture medium within each $\mu$-channel. This initial fixation was followed by complete replacement of pre-fixed medium with fresh PFA solution for 20 min at room temperature. Fixed monolayers were then permeabilised using $0.1 \%$ Triton X-100 in PBS for 10 min. Blocking was achieved by incubating cells for $1 \mathrm{~h}$ with blocking agent containing $3 \%$ bovine serum albumin (Sigma, Germany) and $0.1 \%$ Tween 20 (Sigma, Germany) in PBS. Between each step, cells were washed 3 times using PBS with $\mathrm{Mg}^{2+}$ and $\mathrm{Ca}^{2+}$ (Sigma, Germany).

VE-cadherin staining: Mouse anti-VE-cadherin Alexa Flour 488 conjugated antibody (Invitrogen, Germany) was diluted (1:200) in PBS containing 3\% BSA. Cells were incubated with the staining solution for $1 \mathrm{~h}$ at room temperature. Finally, to visualise the cell nucleus, samples were stained with DAPI (1:1000, Sigma, Germany), and images were captured using a confocal laser scanning microscope (Zeiss, LSM 780, Germany). Using ImageJ software (NIH, USA), the fluorescence intensity of VE-cadherin was quantified for all captured images.

F-actin staining: Actin filaments were labelled with Alexa Flour 633 phalloidin (Thermofisher Scientific, Germany). Briefly, samples were incubated for $1 \mathrm{~h}$ with phalloidin staining solution (1:200) in PBS containing 3\% BSA. Cells were counterstained with DAPI as described above and staining patterns were analysed by confocal microscopy. By applying the OrientationJ plug-in for ImageJ, the alignment of actin filaments toward the flow direction was quantified per image according to the sum of distribution of orientation from $-45^{\circ}$ to $+45^{\circ}$.

\subsection{In vitro cytotoxicity assessment}

The Neutral Red (NR) uptake assay is a colorimetric method providing a quantitative estimation of cell viability. It is very often used as a sensitive and accurate test for biomaterial and drug cytotoxicity. NR is a weak cationic dye that relies on the ability of viable cells to uptake the dye via active transport and to incorporate the NR dye into their lysosome. Hence, a decrease in cell viability results in loss of NR uptake 
within the lysosome. Briefly, for static and dynamic experimental setups, cells treated with iron sucrose were washed 3times with PBS, and the assay procedure was performed according to the manufacturer's instruction (BioVision incorporate, Switzerland). For each experimental condition, sample cultured without iron sucrose was used as the negative control. The positive control was prepared by treating cells with 20 $\mathrm{mM}$ of Doxorubicin (BioVision incorporate, Switzerland) for the static and dynamic culture condition, respectively with the incubation time of $72 \mathrm{~h}$. After $2 \mathrm{~h}$ of incubation with complete culture medium containing neutral red solution, the dye in each $\mu$-channel was then extracted under an acidified condition, and the absorbance was measured using a plate reader at $540 \mathrm{~nm}$.

\subsection{Prussian blue staining}

Prussian blue staining was performed for cells under static and dynamic conditions. After treatment with $1800 \mu \mathrm{M}$ of iron sucrose, cells were washed 3 times with PBS to remove any remaining particles within the $\mu$-channels. Then, cells were fixed with $4 \%$ PFA for $10 \mathrm{~min}$, followed by incubation for $5 \mathrm{~min}$ with $4 \%$ potassium ferrocyanide (Sigma, Germany). Next step to proceed was incubating cells in freshly prepared Perl's Prussian blue reagent (4\% Potassium ferrocyanide/ 12\% $\mathrm{HCl}, 1: 1$ volume ratio) for $30 \mathrm{~min}$ at room temperature. Finally, samples were washed several times with PBS and were counterstained with the neutral fast-red solution (Sigma-Aldrich, Germany) for 15 min to enhance visualisation of samples using light microscopy.

\subsection{Transmission electron microscopy (TEM)}

HUVECs were seeded in the Ibidi $\mu$-slides and the cell culture procedure was followed as described in Sect. 2.2. After $24 \mathrm{~h}$ treatment with $1800 \mu \mathrm{M}$ of iron sucrose nanoparticles, cells in both static and dynamic experimental setup were washed three times with warm PBS and were trypsinised using accutase solution. For each experimental set up, control samples were prepared without iron sucrose treatment. The cell pellet was generated by centrifugation at $200 \mathrm{xg}$ for $5 \mathrm{~min}$. The pellet was then sucked up into a capillary tube (Leica-Microsystems, Germany). Therein, cells were fixed with $3 \%$ glutaraldehyde in $0.1 \mathrm{M}$ sodium cacodylate buffer and were washed with $0.2 \mathrm{M}$ sodium cacodylate buffer. After a post-fixation step using $2 \%$ osmium tetroxide in $0.1 \mathrm{M}$ sodium cacodylate buffer, samples were dehydrated through a graded ethanol series followed by acetone and finally embedded in Epon resin (Sigma-Aldrich, Switzerland). Ultrathin sections were contrasted with $2 \%$ uranyl acetate and lead citrate (Reynolds 1963) before imaged in a Zeiss EM 900 (Carl Zeiss Microscopy GmbH, Germany) at 80 kV.

\subsection{Isolation of RNA and RT-PCR}

For both static and dynamic culture condition, cells were treated for $24 \mathrm{~h}$ with $1800 \mu \mathrm{M}$ of iron sucrose complex. HUVECs without treatment and cells treated with $30 \mathrm{ng} / \mathrm{mL}$ of tumor necrosis factor- $a$ (TNF-a; 4 $\mathrm{h}$ incubation time statically, for static and the dynamic cultures) were prepared as negative and positive control, respectively. Total RNA was extracted using the RNeasy mini kit (Qiagen, Germany). After quantifying nucleic acid concentration of each sample with NanoDrop spectrophotometer (ND1000, ThermoFisher, Germany), $200 \mathrm{ng}$ of total RNA was applied as a template for cDNA synthesis. Primers for VCAM-1, ICAM-1, and GAPDH were all purchased from Microsynth, Switzerland (Table 1), and GAPDH 
was considered as a housekeeping gene. Quantitative real-time PCR was performed using iQ SYBR Green supermix (Bio-Rad, Germany) on the CFX96 real-time PCR detection system (Bio-Rad, Germany). Samples were run in quadruple, and relative expression values were calculated using the $\Delta \Delta \mathrm{c}_{\mathrm{t}}$ method. All samples were tested for the absence of non-specific amplification.

Table 1 Real-time PCR primer sequences used in the experiment.

\begin{tabular}{cll}
\hline Gene & \multicolumn{1}{c}{ Forward } & \multicolumn{1}{c}{ Reverse } \\
\hline VCAM-1 & 5'-TCT ACG CTG ACA ATG AAT CCT G-3' & 5'-AGG GCC ACT CAA ATG AAT CTC-3' \\
ICAM-1 & 5'-CAA TGT GCT ATT CAA ACT GCC C-3' & 5'-CAG CGT AGG GTA AGG TTC TTG-3' \\
GADPH & 5'-AGT CAG CCG CAT CTT CTT TT-3' & 5'-CCA ATA CGA CCA AAT CCG TTG-3' \\
\hline
\end{tabular}

\subsection{Statistical analysis}

Graphs and statistical analysis were perfumed using OriginLab software (Massachusetts, USA). Unless otherwise noted, results are represented as the mean \pm standard error of the mean (SEM). One-way analysis of variances (ANOVA) was used to identify and significant differences between the means if independent groups. Further Bonferroni post hoc test was performed with ANOVA to find means that were significantly different between groups. A p-value $<0.05$ was considered as significant.

\section{Results}

\subsection{HUVECs physiological response to the flow}

Using an advanced in vitro perfusion system, the effect of shear stress on HUVEC morphology was identified during cultivation periods of 2, 7, and 14 days. Overall, by exposing cells to a continuous shear stress of 10 dyne $/ \mathrm{cm}^{2}$, a marked change in cellular morphology was observed using light microscopy. After 2 days of dynamic culture, cells reached the so-called cobblestone appearance with no obvious alignment (Fig. 2-a). By continuing the dynamic condition, the alteration of cell morphology from cobblestone structure to a spindle-like appearance was seen on day 7 (Fig. 2-b). Additionally, in comparison with observations on day 2, cells exhibited a notable response to the flow direction on day 7 . After 14 days, a high level of cell alignment in parallel to the direction of the flow was achieved, and tightly packed cells with spindle-shaped morphologies resembled mature endothelial cells residing in a quiescent phase (Fig. 2-c).

\subsection{Re-organisation of actin filaments in HUVECs monolayer}

Using Phalloidin staining, significant changes in the organisation of actin filaments was revealed by confocal laser scanning microscopy. On day 2 of dynamic perfusion culture, actin filaments were distributed throughout cells in a radial pattern displaying random orientation, and many of the actin 
bundles appeared fragmented along the cell length (Fig. 2-d). When HUVECs were further kept under dynamic culture, a dramatic re-organisation of the filamentous actin was detected on day 7 (Fig. 2-e). Actin stress fibres known as tension-bearing structures play an essential role in reacting to the

surrounding physical environment and remodelling of cell structure. ${ }^{[18]}$ In contrast to day 2 , on day 7 , remodelling of filaments into arrays of stress fibres was also observed. Rearrangement of actin filaments from day 2 to day 7, was further confirmed with quantification of confocal images using the ImageJ software (Fig. 2-g, h). Notably, on day 7, the majority of the stress fibres extended across the length of the cells to the direction of flow. Flow continuation demonstrated uniformly aligned cell in the direction of flow on day 14. Furthermore, adaptation to the flow was achieved after two weeks of dynamic culture with the shear stress of $10 \mathrm{dyne} / \mathrm{cm}^{2}$, leading to a decrease in the number of stress fibres and localisation of actin filaments at the cell periphery (Fig. 2-f, i).

\subsection{VE-cadherin expression and HUVECs viability}

For cells cultured under shear stress, the expression of VE-cadherin as a junctional protein was assessed using immunofluorescence staining. Figure 3 shows an abundant and uniform presence of VE-cadherin localisation at the cell-cell contact areas after two weeks of flow exposure. By day 14, confocal images represented labelled VE-cadherin as a continuous and smooth band following the periphery of the cells, and rarely any gaps between HUVECs monolayer was detected (Fig. 3-c). Quantification of microscopic images also confirmed a 2-fold increase in the average intensity of the VE-cadherin fluorescence signal on day 14 in comparison with day 2 of culture (Fig. 3-d).

During the two-week culture condition, cell viability was measured on day 2, 7, and 14 using a colorimetric assay. As shown in Fig. 3-e, cellular viability was maintained under dynamic culture (shear stress: 10 dyne $/ \mathrm{cm}^{2}$ ). Results indicated that between day 7 and day 14 , there was no statistical difference between the numbers of viable cells. Consistency in cellular number could be another confirmation of achieving HUVECs monolayer in the quiescent stage and therefore reduced cell proliferation.

\subsection{Cell viability after treatment with iron sucrose complex}

The effect of iron sucrose complexes on cell viability was assessed after $24 \mathrm{~h}$ incubation time for both static and dynamic culture conditions. Neutral red viability assay was employed to quantify cell viability in both conditions using a quantitative spectrophotometry approach. As shown in Fig. 3-f, for cells cultivated under dynamic condition, the neutral red absorbance value of $73 \% \pm 11$ (variance) was quantified in comparison to the negative control. On the contrary, when cells were treated with iron sucrose under the static condition, the absorbance was remarkably reduced to $52.7 \% \pm 0.7$ (variance). Since there is a direct correlation of neutral red absorbance with cell viability, results showed that the ability of HUVECs to uptake neutral red dye was significantly reduced $(P$-value $<0.05)$ for cells cultivated under the static condition when compared to dynamic culture.

\subsection{Prussian blue staining for intracellular iron sucrose}


After 5 and $24 \mathrm{~h}$ of incubation with iron sucrose complex $(1800 \mu \mathrm{M})$, strong uptake of particles was noticeable for HUVECs under the static condition as shown in Fig. 3. Contrary to the static experimental setup, HUVECs under dynamic condition exhibited far less intercellular particles. Quantitative observation of images revealed an average total surface area of $22.6 \pm 1.2 \%$ covered with Prussian blue stain under static condition. However, this value was significantly less for cells under dynamic condition $(1.4 \pm 0.1 \%)$. For both culture conditions, particles appeared to be in clusters in the cell cytoplasm and mainly around the nucleus.

\subsection{TEM of HUVECs after iron sucrose incubation}

In addition to the observation of cellular uptake by Prussian blue staining, the investigation of the deposition of iron sucrose particles within HUVECs at a concentration of $1800 \mu \mathrm{M}$ was conducted for both static and dynamic culture conditions using TEM. As shown in Fig. 4a-f, TEM images confirmed the internalisation of iron sucrose particles in HUVECs under static and dynamic condition. For both culture conditions, iron sucrose particles were found to be internalised mainly within the cytoplasm, particularly in numerous endocytic vesicles such as lysosomes (Fig. 4c, f). Notably, iron sucrose aggregates were located within cells, and there was no presence of the particles bounded to the cell surface.

\subsection{Gene expression of VCAM-1 and ICAM-1}

The real-time qPCR analysis showed that iron sucrose- induced VCAM- 1 and ICAM- 1 expression in HUVECs was decreased by cultivating cells under flow condition (Fig. 4). Real-time qPCR data analysis with $\Delta \Delta \mathrm{c}_{\mathrm{t}}$ method revealed that for HUVECs under dynamic culture condition, relative expression of VCAM-1 was significantly reduced from $13.7 \pm 1.5$ to $4.6 \pm 0.7$ ( $p$-value $<0.05$ ) when compared with cells treated with iron sucrose particles under static culture condition. In connection with ICAM-1 gene expression, the same trend was observed. Relative expression values of $2.1 \pm 0.5$ and $17.7 \pm 1.4$ were measured for ICAM-1 under dynamic and static conditions, respectively. For both culture conditions, HUVECs treated with TNF- $\alpha$ at the concentration of $30 \mathrm{ng} / \mathrm{mL}$ (positive control) exhibited a tremendously high gene expression of VCAM-1 and ICAM-1.

\section{Discussion}

In this study, we have investigated whether changes to the standard in vitro setups to more physiological models can facilitate our understanding of nanoparticles behaviour and address the controversies between results from in vitro and in vivo studies. In this work, we characterised the uptake of ironcarbohydrate nanoparticles by endothelial cells while comparing the application of an advanced dynamic culture model with the conventional static culture condition. The effect of the two culture conditions (static versus dynamic) on cellular uptake, endothelium viability and functionality were investigated. Although the effect of shear stress on HUVECs has been studied [13], [19], [20], the impact of shear on nanoparticles uptake and their physiological behaviour has been less explored. Using an advanced in vitro perfusion system, we were able to achieve a mature endothelial monolayer while having control over 
experimental parameters, including shear stress, pressure, and flow rate. Over two weeks of cultivating the primary HUVECs under this dynamic condition at the shear stress of $10 \mathrm{dyne} / \mathrm{cm}^{2}$, cells exhibited characteristics of mature endothelial cells, such as spindle-like morphology (Fig. 2a-c), re-modelling of actin filaments toward the flow direction (Fig. 2d-i), maintained cell viability (Fig. 3b-e) as well as increased expression of VE-cadherin (Fig. 3a-d). For endothelial cells lining the inner surface of veins, the normal range of shear stress can vary between 1 to $15 \mathrm{dyne} / \mathrm{cm}^{2}$ based on vein size. In all experiments throughout this study, shear stress of $10 \mathrm{dyne} / \mathrm{cm}^{2}$ was used to allow comparison with other existing published literature in the field. ${ }^{[21]}$

The determination of HUVECs viability after $24 \mathrm{~h}$ exposure to iron sucrose nanoparticles was the first step in comparing the dynamic and static culture condition. Since the role of lysosome in iron metabolism has been widely established ${ }^{[22]}$, a cytotoxicity test (Neutral red assay) based on lysosomal activity was chosen for this experiment. Also, in comparison to other viability tests, such as lactate dehydrogenase (LDH), MTT, and MTS assays, Neural red was the most compatible assay to be employed considering the geometry of $\mu$-slides. Overall, as shown in Sect. 3.4, the comparison between the two-culture conditions confirmed cells ability to maintain high viability after incubation with iron sucrose particles under dynamic culture condition. As demonstrated in Fig. 3f, HUVECs ability to uptake Neural red dye was significantly decreased under static culture, confirming reduced cell viability, which is in agreement with other publications using conventional static culture. [15], [23]

In this study, the investigation into cellular viability was performed using iron sucrose concentration in accordance with the clinical applied doses, subsequently our viability test reflects the potential misleading results obtained from conventional in vitro systems in comparison to an advanced dynamic culture model to mimic endothelium under physiological shear stress. There are a noticeable number of animal and clinical studies demonstrating the safety and effectiveness of intravenous iron sucrose for

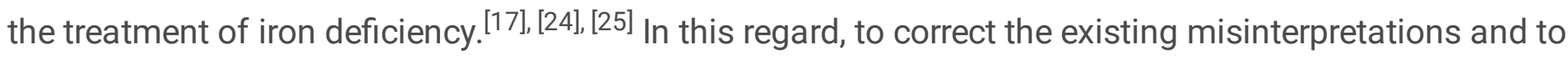
minimise conflicts between in vitro and in vivo reports, efforts should be made to utilise in vitro models that to a great extent mimic the physiological conditions, represent cell-cell interactions, and are providing high sensitivity and accuracy when used to investigate nanoparticles behaviour and stability. ${ }^{[7], ~[26] ~}$

To determine the impact of shear stress on cellular uptake of iron sucrose nanoparticles by HUVECs, Perl's Prussian blue staining method ${ }^{[27]}$ was implemented for both dynamic and static culture condition. Microscopic images, in combination with quantitative image analysis, revealed a remarkable difference between the two culture states. In contrary to results for static culture, a smaller number of particles were taken up by cells under flow-associated shear stress (Fig. 3g-j). To determine whether the observed cellular response is dependent on the physicochemical properties of nanoparticles, the same experimental setup was used for fluorescently labelled polystyrene nanoparticle ( $50 \mathrm{~nm}$ in diameter). The same trend was observed for HUVECs after $24 \mathrm{~h}$ exposure to polystyrene particles as depicted by the confocal microscopy images (supplementary 1 ). Further, to differentiate between cell surface-bound and internalised iron sucrose particles, in addition to Perl's staining, TEM images confirmed the cellular 
internalisation of iron sucrose particles in both static and dynamic culture conditions (Fig. 4a-f, supplementary 2).

Further investigation into differences between HUVECs response to iron sucrose particles under static and dynamic in vitro model was performed by measuring the gene expression level of VCAM- 1 and ICAM1using real-time qPCR. VCAM-1 and ICAM-1 encode cell surface glycoproteins that are predominantly expressed in endothelial cells. ${ }^{[28]}$ Studies have shown that the expression of VCAM-1 and ICAM-1 can be activated during inflammatory stimulation with pro-inflammatory cytokines including TNF-a (used in our investigations as a positive control).[29], [30] Previous studies suggested that nanoparticles with small sizes $<200 \mathrm{~nm}$ can play an important role in the initiation of inflammation and the subsequent penetration of particles though the inflamed region of the vascular system. ${ }^{[26]}$ The results from real-time PCR confirmed a significant increase in the expression of both VCAM- 1 and ICAM- 1 genes after $24 \mathrm{~h}$ of incubation with iron sucrose particles in static condition. Although, the expression of both genes was noticed in dynamic samples, the relative expression level was significantly lower in comparison to the conventional static culture (Fig. 4g-j). Notably, light micrograph images captured using Prussian blue staining assay also revealed the disruption of the cell monolayer (showed with arrows in Fig. 3h) when compared with HUVECs morphology under dynamic condition exhibiting intact cell-cell contacts (Fig. 3j).

\section{Conclusions}

Significant differences in the cellular behaviour of endothelial cells under flow exposure have been exhibited during long-term in vitro culture. Flow exposure to HUVECs under physiological shear stress induced remarkable cell structure remodelling and elongation toward the flow direction after two weeks of cultivation. In contrast to static culture, cells viability and functionality in dynamic culture condition found to be highly maintained, and cell exhibited less sensitivity to iron sucrose nanoparticles. Cellular uptake of iron sucrose particles was significantly reduced when dynamic culture condition was employed compared to static cultures. Overall, the different cellular response under flow conditions demonstrated outcomes remarkably differed from cellular responses under static condition. Our investigation strengthened the evidence that dynamic cell culture condition can provide a better resemblance to endothelial cell behaviour in vivo. The role of -physiologically relevant in vitro models will be increasingly important in the future of nanotechnology in medicine. The development of advanced cell culture models with great potential in mimicking in vivo characteristics of specific cell types can improve our understanding of cellular response to nanoparticles and can provide a robust platform for the toxicological observation of injectable nanoparticles. Further advances in dynamic in vitro models with the implementation of different cell types, can potentially pave the way for reducing the need for costly animal studies.

\section{Declarations}

\section{Authors contribution}


NN designed, conducted experiments and prepared the original manuscript. LD (TEM) SC (PCR) and AR (cell culture) supported the execution of the experimental work. PW designed together with NN the study and revised the manuscript. All authors were engaged in commenting on the manuscript.

\section{Acknowledgements}

N.N. acknowledges the EMPAPOSTDOCS-II program, which has received funding from the European Union's Horizon 2020 research and innovation program under the Marie Skłodowska-Curie grant agreement number 754364. Open Access funding provided by Lib4RI - Library for the Research Institutes within the ETH Domain: Eawag, Empa, PSI \& WSL.

\section{Data availability.}

The datasets generated during and/or analysed during the current study are available from the corresponding author on reasonable request.

\section{Conflict of interest}

Authors declare that there is no conflict of interest.

\section{References}

1. Tinkle, S. et al. Nanomedicines: Addressing the scientific and regulatory gap. Ann. N. Y. Acad. Sci. 1313 (no. 1), 35-56 https://doi.org/10.1111/nyas.12403 (2014).

2. Hafner, A., Lovrić, J., Lakoš, G. P. \& Pepić, I. Nanotherapeutics in the EU: an overview on current state and future directions. Int. J. Nanomedicine. 9, 1005 (2014).

3. Soares, S., Sousa, J., Pais, A. \& Vitorino, C. Nanomedicine: Principles, Properties, and Regulatory Issues. Front. Chem. 6, 360 https://doi.org/10.3389/fchem.2018.00360 (2018).

4. Flühmann, B., Ntai, I., Borchard, G., Simoens, S. \& Mühlebach, S. Nanomedicines: The magic bullets reaching their target? Eur. J. Pharm. Sci. Off. J. Eur. Fed. Pharm. Sci. 128, 73-80 https://doi.org/10.1016/j.ejps.2018.11.019 (Feb. 2019).

5. Nikravesh, N. et al. Factors influencing safety and efficacy of intravenous iron-carbohydrate nanomedicines: From production to clinical practice. Nanomedicine Nanotechnology, Biol. Med. 26, 102178 https://doi.org/10.1016/j.nano.2020.102178 (2020).

6. Polson, A. G. \& Fuji, R. N. The successes and limitations of preclinical studies in predicting the pharmacodynamics and safety of cell-surface-targeted biological agents in patients. Br. J. Pharmacol. 166 (no. 5), 1600-1602 (2012). 
7. Mahmoudi, M. Debugging Nano-Bio Interfaces: Systematic Strategies to Accelerate Clinical Translation of Nanotechnologies. Trends Biotechnol. 36 (no. 8), 755-769 https://doi.org/10.1016/j.tibtech.2018.02.014 (2018).

8. Freese, C. et al. In vitro investigation of silica nanoparticle uptake into human endothelial cells under physiological cyclic stretch. Part. Fibre Toxicol. 11 (no. 1), 1-12 https://doi.org/10.1186/s12989014-0068-y (2014).

9. Fröhlich, E. "Comparison of conventional and advanced in vitro models in the toxicity testing of nanoparticles," Artif. Cells, Nanomedicine Biotechnol., vol. 46, no. sup2, pp. 1091-1107 2018, doi: 10.1080/21691401.2018.1479709.

10. Cines, B. D. B. et al. "The Journal of The American Society of Hematology," vol. 91, no. 10, pp.35273561, 2019.

11. Aird, W. C. Spatial and temporal dynamics of the endothelium. J. Thromb. Haemost. 3 (no. 7), 13921406 https://doi.org/10.1111/j.1538-7836.2005.01328.x (2005).

12. Jiménez, N., Krouwer, V. J. D. \& Post, J. A. A new, rapid and reproducible method to obtain high quality endothelium in vitro. Cytotechnology. 65 (no. 1), 1-14 https://doi.org/10.1007/s10616-0129459-9 (2013).

13. Malek, A. M. \& Izumo, S. Mechanism of endothelial cell shape change and cytoskeletal remodeling in response to fluid shear stress. J. Cell Sci. 109 (no. 4), 713-726 (1996).

14. Borchard, G., Flühmann, B. \& Mühlebach, S. Nanoparticle iron medicinal products - Requirements for approval of intended copies of non-biological complex drugs (NBCD) and the importance of clinical comparative studies. Regul. Toxicol. Pharmacol. 64 (no. 2), 324-328 https://doi.org/10.1016/j.yrtph.2012.08.009 (2012).

15. Kamanna, V. S., Ganji, S. H., Shelkovnikov, S., Norris, K. \& Vaziri, N. D. Iron sucrose promotes endothelial injury and dysfunction and monocyte adhesion/infiltration. Am. J. Nephrol. 35 (no. 2), 114-119 https://doi.org/10.1159/000334939 (2012).

16. Carlini, R. G., Alonzo, E., Bellorin-Font, E. \& Weisinger Jose, J. R. R., "Apoptotic stress pathway activation mediated by iron on endothelial cells in vitro. Nephrol. Dial. Transplant. 21 (no. 11), 30553061 https://doi.org/10.1093/ndt/gfl341 (2006).

17. Praschberger, M. et al. Bioavailability and stability of intravenous iron sucrose originator versus generic iron sucrose AZAD. Pharm. Dev. Technol. 20 (no. 2), 176-182 https://doi.org/10.3109/10837450.2013.852575 (2015).

18. Burridge, K. \& Wittchen, E. S. The tension mounts: Stress fibers as force-generating mechanotransducers. J. Cell Biol. 200 (no. 1), 9-19 https://doi.org/10.1083/jcb.201210090 (2013).

19. Ukropec, J. A., Hollinger, M. K. \& Woolkalis, M. J. Regulation of VE-cadherin linkage to the cytoskeleton in endothelial cells exposed to fluid shear stress. Exp. Cell Res. 273 (no. 2), 240-247 https://doi.org/10.1006/excr.2001.5453 (2002).

20. Biology, M. "Model for the Alignment of Actin Filaments," vol. 59, no. 6, pp.1029-1046, 1997. 
21. Papaioannou, T. G. \& Stefanadis, C. Vascular wall shear stress: Basic principles and methods. Hell. J. Cardiol. 46 (no. 1), 9-15 (2005).

22. Kurz, T., Eaton, J. W. \& Brunk, U. T. The role of lysosomes in iron metabolism and recycling. Int. J. Biochem. Cell Biol. 43 (no. 12), 1686-1697 https://doi.org/10.1016/j.biocel.2011.08.016 (2011).

23. Praschberger, M. et al. "Iron sucrose and ferric carboxymaltose: no correlation between physicochemical stability and biological activity," Biometals, vol. 28, no. 1, pp.35-50, 2015.

24. Cançado, R. D., de Figueiredo, P. O. N., Olivato, M. C. A. \& Chiattone, C. S. Efficacy and safety of intravenous iron sucrose in treating adults with iron deficiency anemia. Rev. Bras. Hematol. Hemoter. 33 (no. 6), 439-443 https://doi.org/10.5581/1516-8484.20110119 (2011).

25. Tomer, A. et al. "The safety of intravenous iron preparations: Systematic review and meta-analysis," Mayo Clin. Proc., vol. 90, no. 1, pp. 12-23 2015, doi: 10.1016/j.mayocp.2014.10.007.

26. Khodabandehlou, K., Masehi-Lano, J. J., Poon, C., Wang, J. \& Chung, E. J. Targeting cell adhesion molecules with nanoparticles using in vivo and flow-based in vitro models of atherosclerosis. Exp. Biol. Med. 242 (no. 8), 799-812 https://doi.org/10.1177/1535370217693116 (2017).

27. Wu, X., Tan, Y., Mao, H. \& Zhang, M. Toxic effects of iron oxide nanoparticles on human umbilical vein endothelial cells. Int. J. Nanomedicine. 5 (no. 1), 385-399 https://doi.org/10.2147/ijn.s10458 (2010).

28. Liao, J. K. \& Liao, J. K. "Linking endothelial dysfunction with endothelial cell activation Find the latest version: Linking endothelial dysfunction with endothelial cell activation," vol. 123, no. 2, pp.540-541, 2013, doi: $10.1172 / \mathrm{JCl} 66843.540$.

29. Kong, D. H., Kim, Y. K., Kim, M. R., Jang, J. H. \& Lee, S. Emerging roles of vascular cell adhesion molecule-1 (VCAM-1) in immunological disorders and cancer. Int. J. Mol. Sci. 19 (no. 4), 13-17 https://doi.org/10.3390/ijms19041057 (2018).

30. Frank, P. G. \& Lisanti, M. P. "ICAM-1: role in inflammation and in the regulation of vascular permeability.," American journal of physiology. Heart and circulatory physiology, vol. 295, no. 3. pp. H926-H927, Sep 2008, doi: 10.1152/ajpheart.00779.2008.

\section{Figures}


Detaching cells with Seeding cells in a $\mu$-channel slide Cultivating cells under flow Accutase coated with collagen

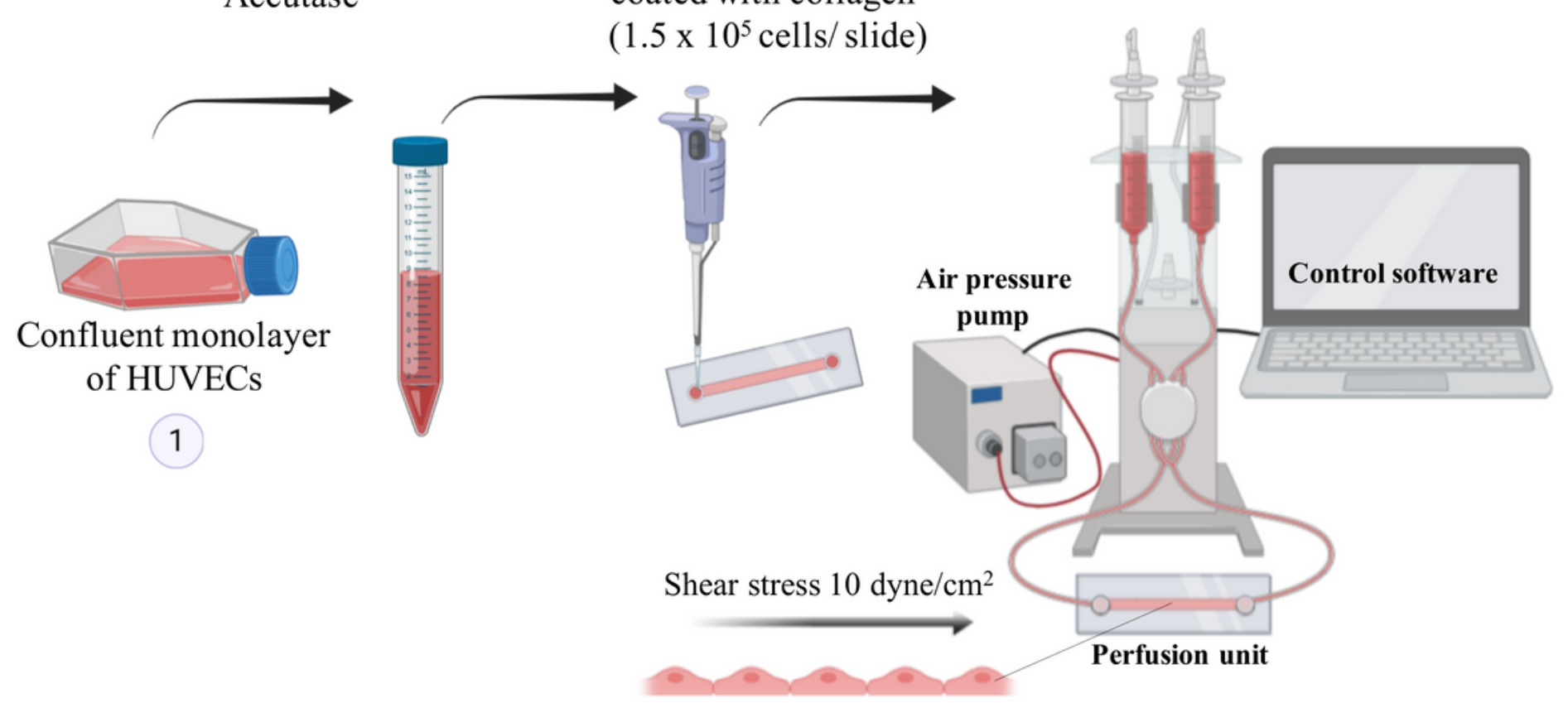

\section{Figure 1}

Schematic illustration showing the preparation procedure for the dynamic cultivation of primary HUVECs using an advanced semi-automated perfusion system. Illustration created with Biorender.com. 
a

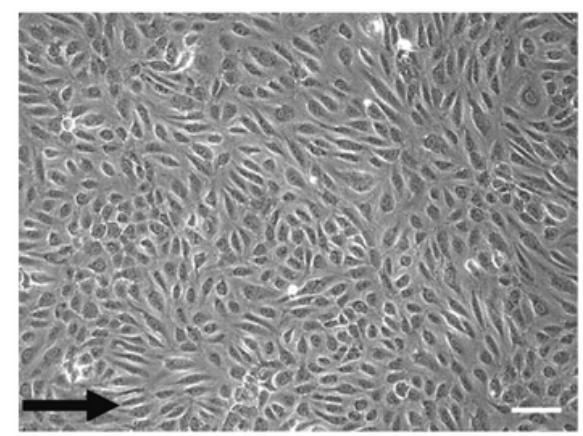

d
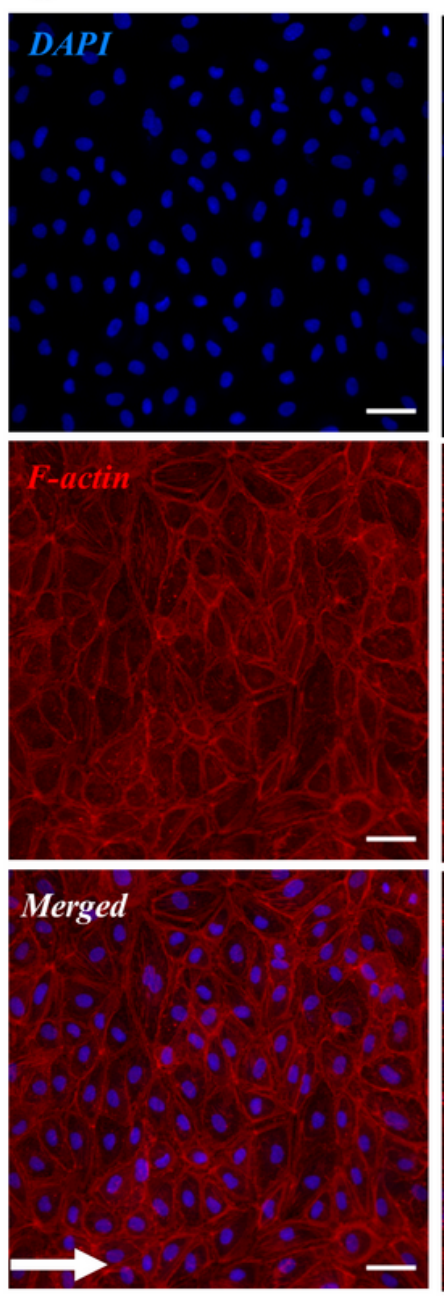

b

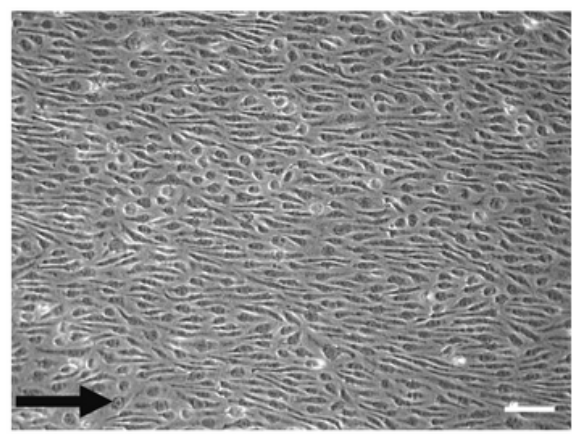

f
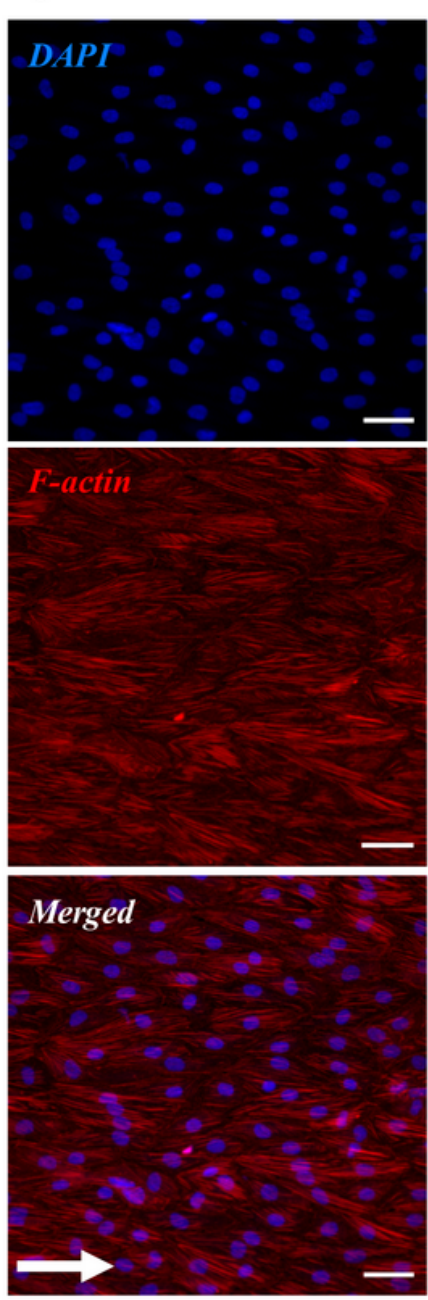
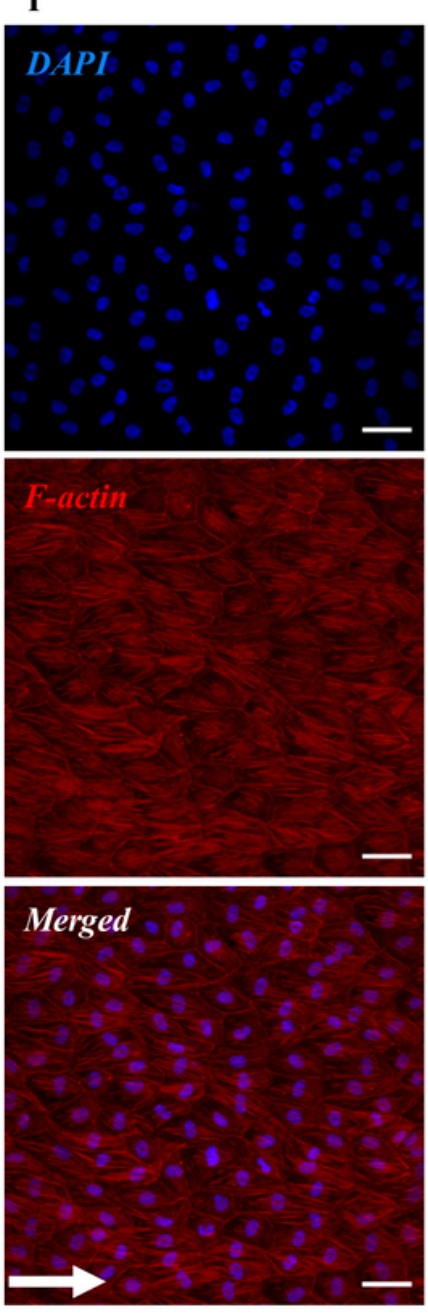

c

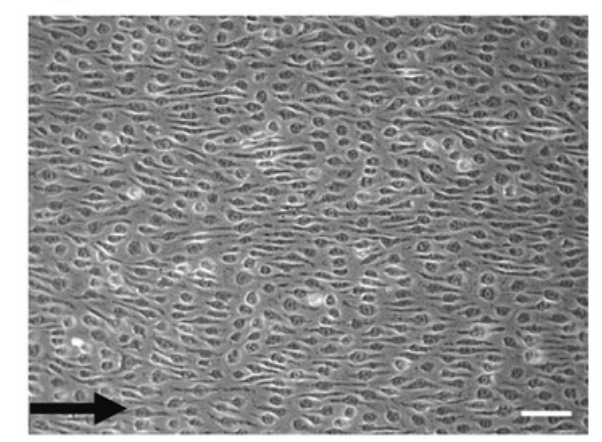

g
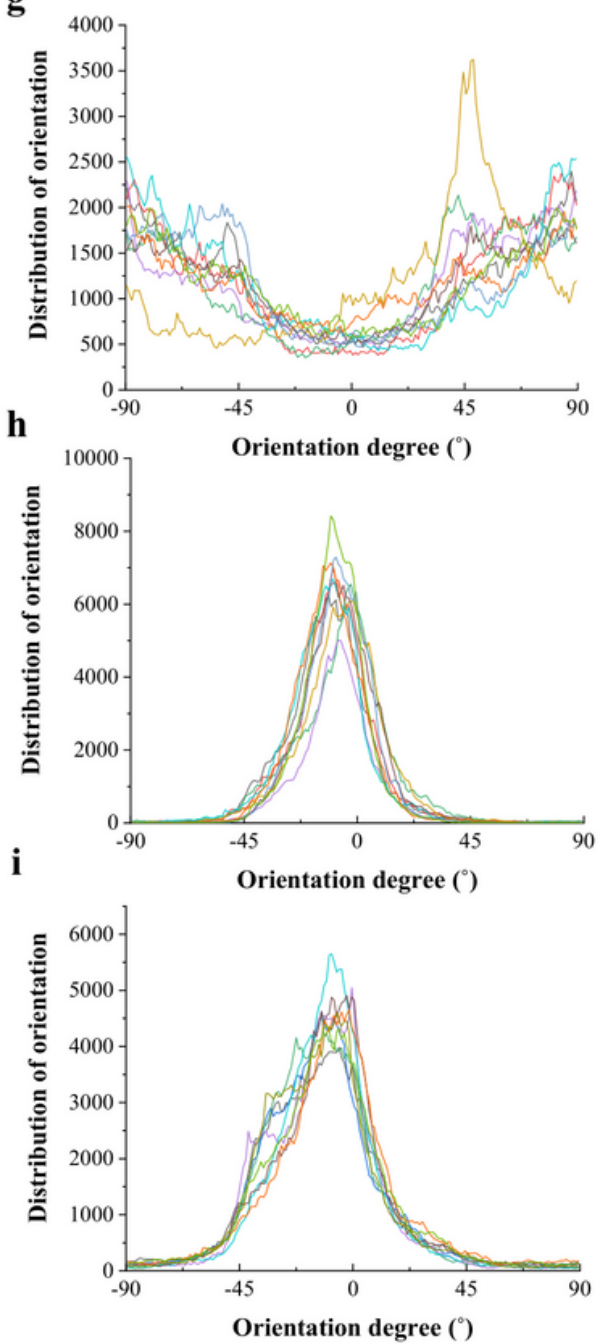

Figure 2

Observation of HUVECs response to continuous unidirectional flow at 10 dyne /cm2 shear stress for cultivation time of up to two weeks. a-c light microscopic images showed HUVECs morphological change after 2,7 , and 14 days of dynamic culture, respectively. d-f confocal microscopy images confirmed the remodelling of actin filaments after exposure to shear stress on day 2, 7, and 14 days, respectively (RED: actin filaments, BLUE: cell nucleus). g-i Distribution of actin filaments toward the flow direction was quantified using ImageJ software on day 2, 7, and 14, respectively (different colours in each diagram 
related to the orientation of actin filaments per each captured image). The arrows indicate the direction of flow. Scale bars: $40 \mu \mathrm{m}$.

a

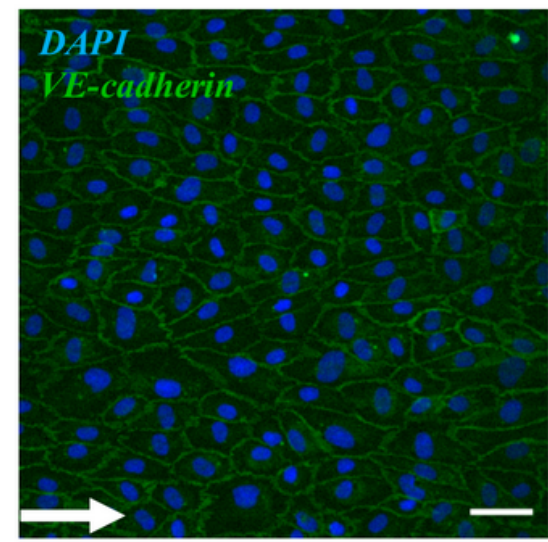

d

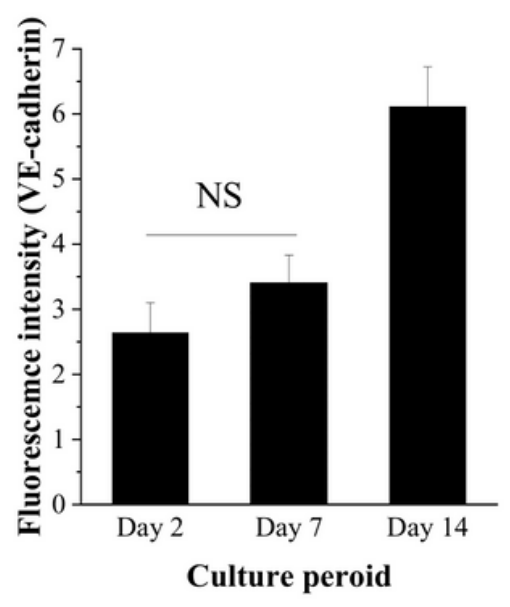

b

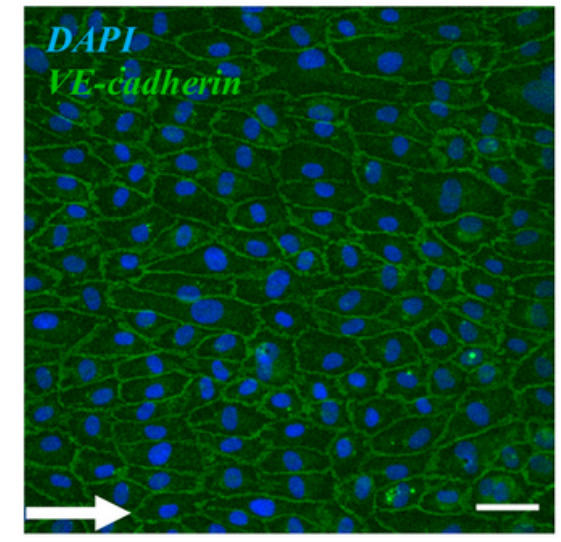

e

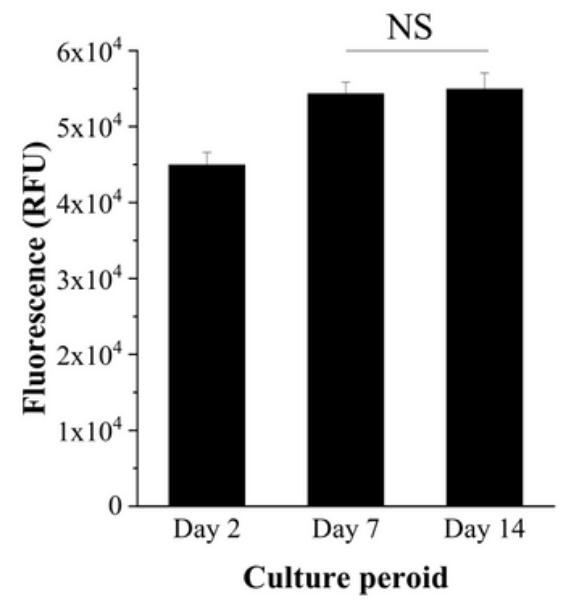

i

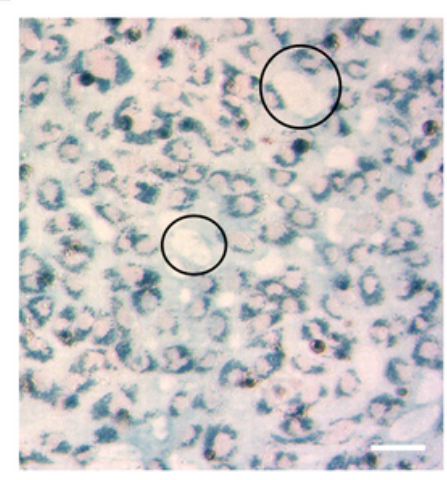

c

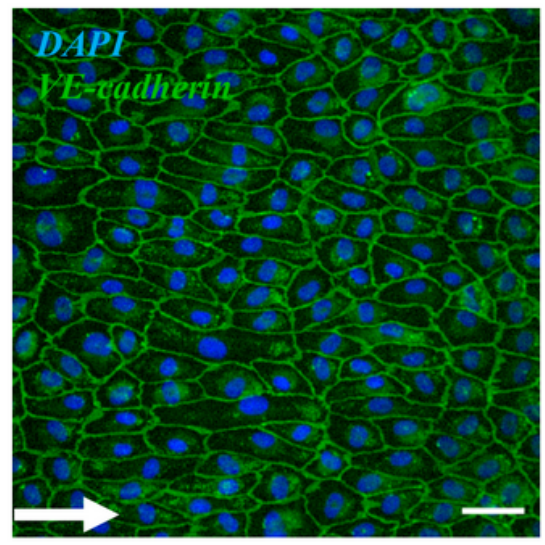

f

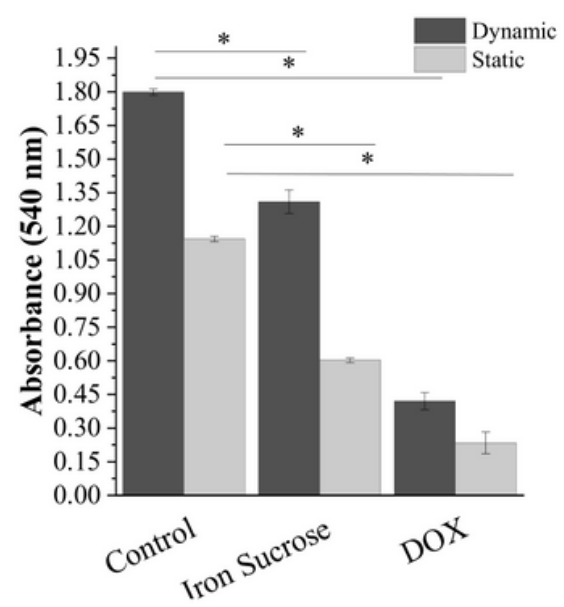

j

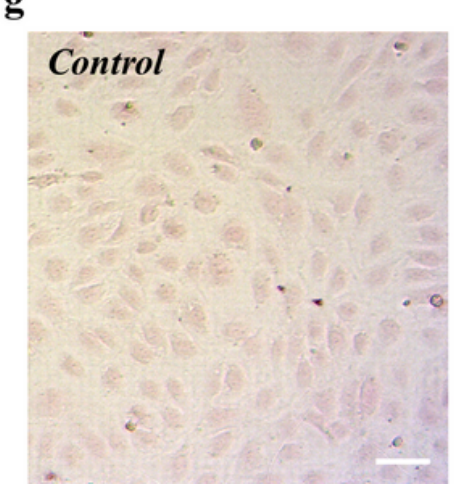

h 
sucrose complex effect on cell viability after $24 \mathrm{~h}$ exposure time in a static (conventional method) versus dynamic condition. g-j Prussian blue stained and nucleus fast red counterstained HUVECs incubated with $1800 \mu \mathrm{M}$ of iron sucrose complex for $24 \mathrm{~h}$; Static condition: $\mathrm{g}$ (without treatment) and $\mathrm{h}$ (with treatment, circles show disruption of the HUVECs monolayer); dynamic condition: $i$ (without treatment) and $j$ (with treatment). Scale bars: $40 \mu \mathrm{m}$, (P-value $<0.05)$.

a

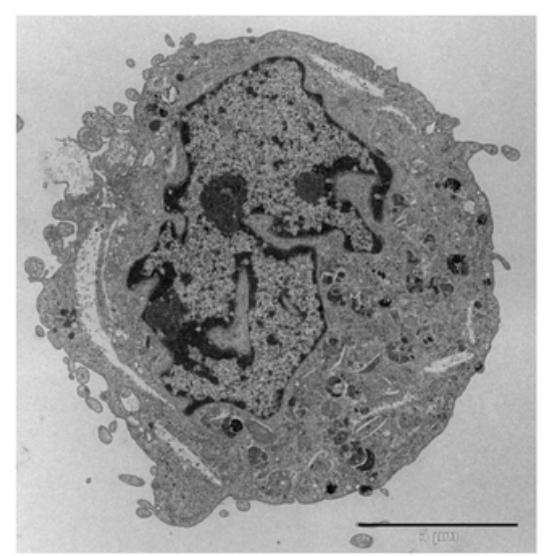

d

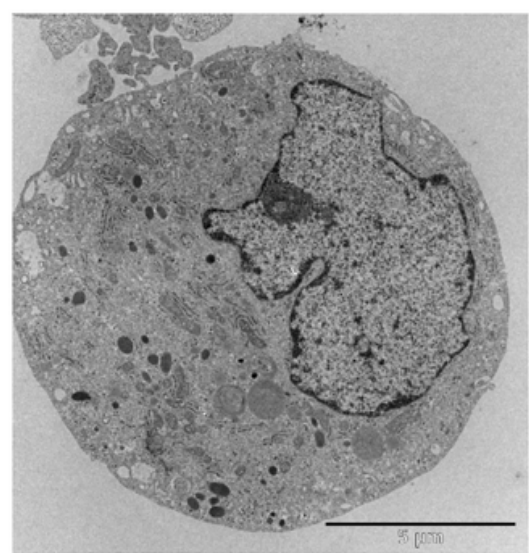

g

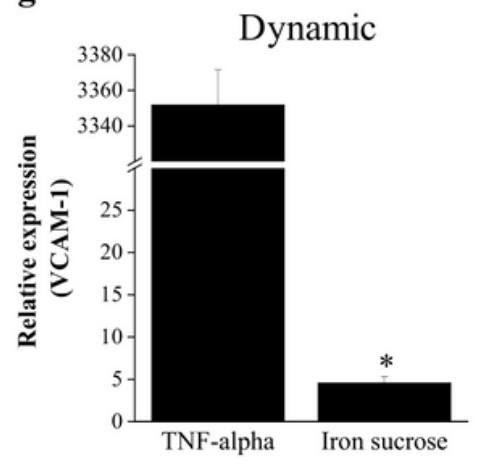

h b

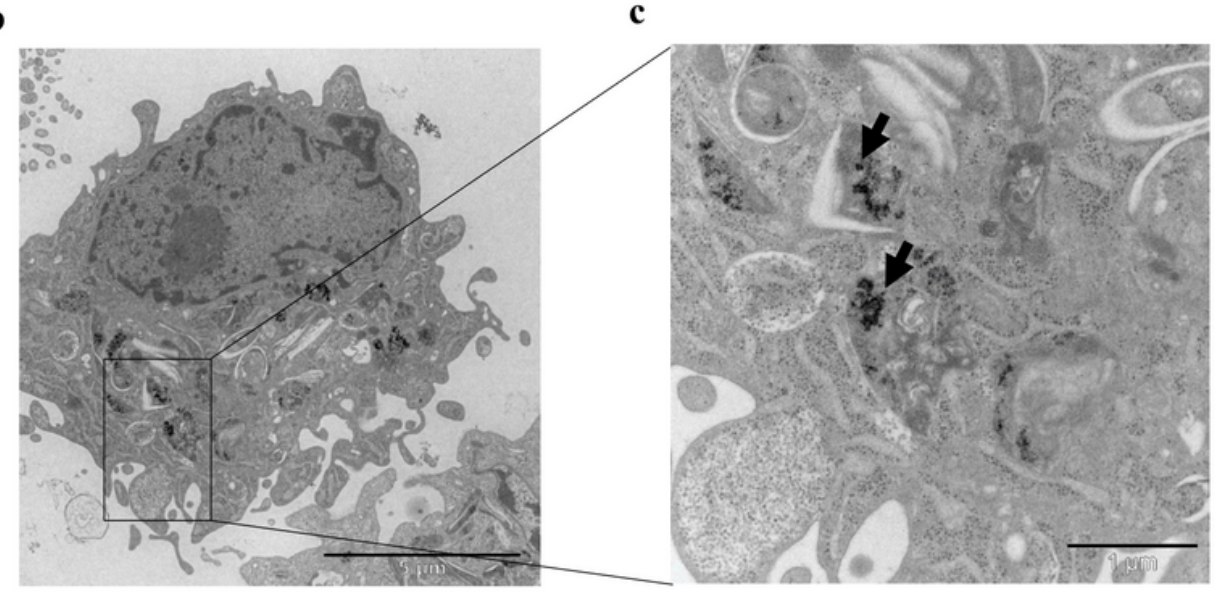

e

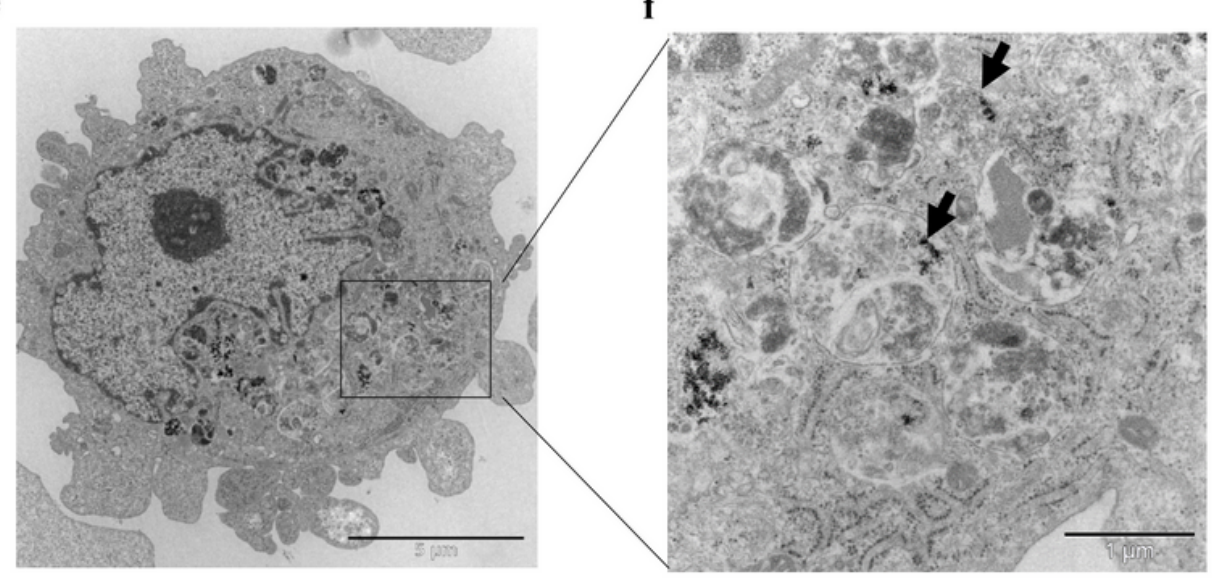

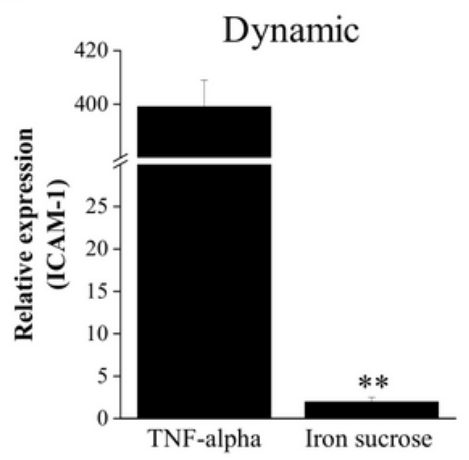

i

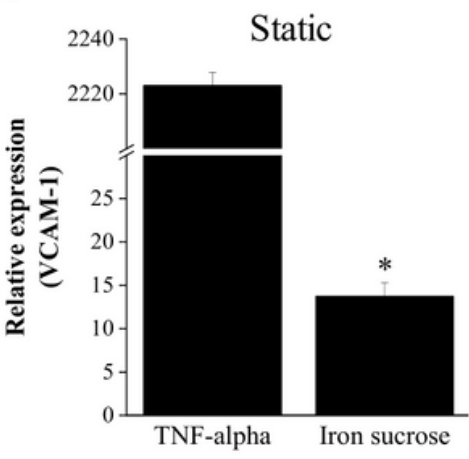

j

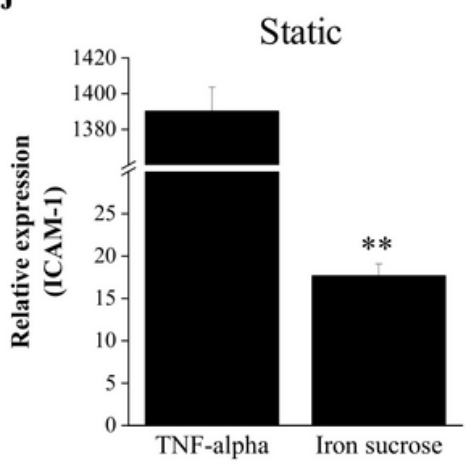

Figure 4

a-f Cellular uptake of iron sucrose complex in HUVECs after $24 \mathrm{~h}$ incubation time, evaluated by TEM (a-c: dynamic culture, d-f: static culture). The particles were mainly internalised in the cytoplasmic vesicles (black arrows in $c, f)$, and no iron particles in the negative controls $(a, d)$ were observed. Panel $c$ and $f$ are magnified images of the rectangular area of panels $b$ and e. Scale bars: $5 \mu \mathrm{m}(a, b, d, e)$ and $1 \mu \mathrm{m}(c, f) . g-$ 
j Quantitative real-time PCR of VCAM-1 (g: dynamic condition, i: static condition) and ICAM-1 (h: dynamic, j: static) mRNA expression by HUVECs stimulated with TNF-a $(30 \mathrm{ng} / \mathrm{mL}$ for $4 \mathrm{~h})$ or treated with iron sucrose complex $(1800 \mu \mathrm{M}$ for $24 \mathrm{~h})$; $\left({ }^{*}, * *\right.$ P-values $\left.<0.05\right)$.

\section{Supplementary Files}

This is a list of supplementary files associated with this preprint. Click to download.

- GA.png

- SupplementaryManuscriptNikravesh.docx 